\title{
EL CONTROL JUDICIAL DE LA POLÍTICA EXTERIOR: HACIA LA NORMALIZACIÓN DE LA PESC EN EL ORDENAMIENTO JURÍDICO DE LA UNIÓN EUROPEA (A PROPÓSITO DEL ASUNTO BANK REFAH KARGARAN)
}

JUAN SANTOS VARA ${ }^{1}$

savajuan@usal.es

Cómo citar/Citation

Santos Vara, J. (2021).

El control judicial de la política exterior: hacia la normalización de la PESC en el ordenamiento jurídico de la Unión Europea (a propósito del asunto Bank Refah Kargaran). Revista de Derecho Comunitario Europeo, 68, 159-184. doi: https://doi.org/10.18042/cepc/rdce.68.05

\section{Resumen}

La sentencia del TJUE en el asunto Bank Refah Kargaran va a contribuir significativamente a reforzar la protección jurídica de los particulares en relación con las medidas restrictivas adoptadas por la UE. Por primera vez, el TJUE se ha declarado competente para conocer de los recursos que tienen por objeto obtener una indemnización por los perjuicios sufridos como consecuencia de la adopción de medidas restrictivas en el ámbito de la PESC. La sentencia Bank Refah Kargaran tiene profundas implicaciones constitucionales para el ordenamiento jurídico de la UE, ya que viene a confirmar que el rol del TJUE en la PESC no es tan limitado como aparentan los Tratados. Los actos jurídicos de la PESC no pueden ser considerados como instrumentos de naturaleza netamente intergubernamental que se mantienen al margen del control

1 Profesor Titular de Derecho Internacional Público y Relaciones Internacionales y Titular de la Cátedra Jean Monnet en Acción Exterior de la Unión Europea, Universidad de Salamanca. Este trabajo ha sido realizado en el marco del proyecto de investigación «La externalización e informalización de las políticas de inmigración, asilo y gestión de fronteras de la UE: nuevos desafíos jurídicos» (RTI2018-099097-B-I00), financiado por el Ministerio de Ciencia, Innovación y Universidades del que el autor es investigador principal. El autor agradece a los evaluadores anónimos sus comentarios. 
judicial. Los actos de la PESC han de ser interpretados en el marco de la estructura general de los Tratados, incluyendo el sistema general de recursos de la UE.

\section{Palabras clave}

Política Exterior y de Seguridad Común; competencia del TJUE; medidas restrictivas contra personas físicas o jurídicas; responsabilidad extracontractual; recurso de indemnización; sanciones; art. 215 TFUE; art. 47 de la Carta de Derechos Fundamentales.

\section{THE JUDICIAL CONTROL OF FOREIGN POLICY: TOWARDS THE NORMALISATION OF CFSP IN THE EU LEGAL ORDER (WITHIN THE CONTEXT OF THE BANK REFAH KARGARAN CASE)}

\section{Abstract}

The judgement delivered by the Court of Justice of the European Union (CJEU) in Bank Refah Kargaran case is going to substantially improve the judicial protection of persons or entities subject to restrictive measures. The CJEU has accepted for the first time its jurisdiction to rule on the non-contractual liability of the Union as regards actions for damages relating to restrictive measures adopted in the framework of Common Foreign and Security Policy (CFSP). The judgement Bank Refah Kargaran has major constitutional implications for the EU legal order because it confirms that the role of the CFEU in CFSP is not as limited as reflected in the Treaties. CFSP legal acts can not be considered as intergovernmental instruments that are immune to judicial control. CFSP legal acts have to be interpreted in the context of the structure of the Treaties, including the EU system of remedies.

\section{Keywords}

Common Foreign and Security Policy; jurisdiction of the Court; restrictive measures; non-contractual liability; action for damages; restrictive measures; sanctions; article 29 TEU; art. 215 TFEU; art. 47 of the Charter of Fundamental Rights of the European Union.

\section{LE CONTRÔLE JURIDICTIONNEL DE LA POLITIQUE ÉTRANGÈRE: VERS LA NORMALISATION DE LA PESC DANS L'ORDRE JURIDIQUE DE L'UNION EUROPÉENNE (CONCERNANT L'AFFAIRE BANK REFAH KARGARAN)}

\section{Résumé}

L'arrêt de la CJUE dans l'affaire Bank Refah Kargaran va contribuer de manière effective à renforcer la protection juridique des particuliers concernant les mesures 
restrictives adoptées par l'UE. Pour la première fois, la CJUE s'est déclarée compétente pour connaitre les recours ayant pour objet l'obtention d'une indemnisation pour les dommages encourus en raison de l'adoption de mesures restrictives dans le cadre de la PESC. L'arrêt Bank Refah Kargaran a de profondes implications constitutionnelles pour l'ordre juridique de l'UE, étant donné qu'il confirme que le rôle de la CJUE dans la PESC n'est pas aussi limité qu'il le semble à travers les Traités. Les actes juridiques de la PESC ne peuvent être considérés comme des instruments de nature expressément intergouvernementale qui restent en marge du contrôle juridictionnel. Les actes de la PESC doivent être interprétés dans le cadre de la structure générale des Traités, y compris le système de recours de l'UE.

\section{Mots clés}

Polítique Étrangère et de Securité Commun; Compétence de la Cour; mesures restrictives; responsabilité non contractuelle; Recours en indemnité; sanctions; article 29 TUE; art. 215 TFUE; art. 47 de la charte des droits fondamentaux de l'Union européenne. 


\section{SUMARIO}

I. INTRODUCCIÓN. II. LA EXCLUSIÓN DEL CONTROL JUDICIAL DE LA PESC EN LOS TRATADOS. III. EL ALCANCE DEL CONTROL JUDICIAL DE LA PESC EN LA JURISPRUDENCIA DEL TRIBUNAL DE JUSTICIA. IV. LA OPINION DEL ABOGADO GENERAL HOGAN: LA NECESARIA COHERENCIA DE LOS TRATADOS. V. EL ENFOQUE DEL TRIBUNAL DE JUSTICIA: UN AVANCE REVOLUCIONARIO: 1. EI Estado de derecho y la tutela judicial efectiva: una referencia previsible. 2. La relevancia del recurso de indemnización en la PESC y la tutela judicial efectiva. 3. La coherencia del sistema de tutela judicial: un argumento poco convincente. 4. La inaplicación de la jurisprudencia Segi y Gestoras Pro-Amnistía. 5. La dificultad para obtener una reparación por los periuicios sufridos en el ordenamiento jurídico de la UE. VI. REFELEXIONES FINALES. Biblografía.

\section{INTRODUCCIÓN}

El alcance de la competencia del Tribunal de Justicia de la UE (TJUE) para ejercer el control judicial de la Política Exterior y de Seguridad Común (PESC) es un campo que ha permanecido inexplorado hasta hace pocos años. No es sorprendente que no se otorgara un rol específico a los jueces de la UE cuando se estableció la PESC en el Tratado de Maastricht. Se consideraba que las materias que son objeto de la política exterior no podían ser objeto de control judicial porque afectan a cuestiones muy sensibles desde la perspectiva política. Esta situación ha cambiado paulatinamente a medida que la PESC ha ido ampliando sus objetivos y las decisiones adoptadas en el marco de la PESC han tenido implicaciones negativas para los derechos fundamentales (Denza, 2002: 2).

La entrada en vigor del Tratado de Lisboa en 2009 ha modificado sustancialmente el marco constitucional en el que se desarrolla la política exterior. El Tratado de Lisboa abolió la estructura en pilares de la UE y estableció por primera vez un conjunto de principios y valores en el art. 21 TUE que han de guiar el conjunto de la acción exterior. Sin embargo, los Estados miembros han continuado presentando la PESC como una política que no ha superado el carácter intergubernamental de la antigua Cooperación Política Europea "while neglecting an integrationist undercurrent that is boosted by internal 
and external developments» (Wessel, 2016: 440). Es precisamente en este contexto en el que se ha planteado y examinado con particular intensidad el rol del TJUE en el control judicial de la PESC (Andrés Sáenz de Santamaría, 2017: 875; Koutrakos, 2018: 2). En el controvertido Dictamen 2/13 sobre la adhesión de la UE al Convenio de Derechos Humanos (CEDH), el TJUE afirmó que los actos adoptados en el marco de la PESC están generalmente excluidos del control judicial y que «el Tribunal de Justicia aún no ha tenido ocasión de precisar el alcance de las limitaciones de su competencia resultantes, en materia de PESC, de dichas disposiciones» ${ }^{2}$. Esta afirmación dejó completamente abierto el alcance de la competencia del Tribunal para controlar los actos PESC.

Bank Refah Kargaran ha resultado afectado por las medidas restrictivas establecidas por la UE para compeler a Irán a poner fin a su programa de armas nucleares. El 6 de octubre de 2020, el TJUE se pronunció sobre el recurso de casación interpuesto por Bank Refah Kargaran contra la sentencia del Tribunal General (TG) de 10 de diciembre de $2018^{3}$. El TG había llegado a la conclusión de que no era competente para pronunciarse sobre la pretensión de reparación del perjuicio supuestamente sufrido como consecuencia de las medidas restrictivas establecidas en decisiones adoptadas en el marco de la PESC ${ }^{4}$. Previamente, el TG había anulado las medidas restrictivas impuestas al recurrente mediante las decisiones 2010/644 y 2011/783, así como los reglamentos $861 / 2010$ y 267/2012 por el incumplimiento de la obligación de motivación ${ }^{5}$. Posteriormente, el nombre del recurrente fue incluido nueva-

2 Dictamen del Tribunal de Justicia, 2/13, de 18 de diciembre de 2014, EU:C: 2014:2454, apdo. 251.

3 Sentencia de 10 de diciembre de 2018, Bank Refah Kargaran/Consejo, T-552/15, EU:T: 2018:897.

4 Sentencia del Tribunal General de 10 de diciembre de 2018, T-552/15, Bank Refah Kargaran, EU:T:2018:897.

5 Sentencia del Tribunal General de 6 de septiembre de 2013, T-24/11, Bank Refah Kargaran EU:T:2013:403. El TG anuló los siguientes actos jurídicos: Decisión 2010/644/PESC del Consejo, de 25 de octubre de 2010 por la que se modifica la Decisión 2010/413/PESC relativa a la adopción de medidas restrictivas contra Irán y se deroga la Posición Común 2007/140/PESC (DO L 281, de 27.10.2010, p. 81); Decisión 2011/783/PESC del Consejo de 1 de diciembre de 2011 por la que se modifica la Decisión 2010/413/PESC relativa a la adopción de medidas restrictivas contra Irán (DO L 319, de 2.12.2011, p. 71); Reglamento n. ${ }^{\circ}$ 861/2010 de la Comisión, de 5 de octubre de 2010 , por el que se modifica el anexo I del Reglamento (CEE) n. ${ }^{\circ}$ 2658/87 del Consejo, relativo a la nomenclatura arancelaria y estadística y al arancel aduanero común (DO L 284, 29.10.2010, p. 1); Reglamento no 267/2012 del 
mente en la lista de medidas restrictivas a través de la Decisión 2013/661 y el Reglamento 1154/2013 . Se interpuso un segundo recurso de anulación contra estas medidas que fue desestimado por el $\mathrm{TG}^{7}$.

El TG estimó que la recurrente no había distinguido en su recurso entre la responsabilidad de la Unión derivada de las decisiones PESC y de los reglamentos adoptados para implementar las medidas restrictivas. El TG alegó que dispone de una competencia limitada en el ámbito de la PESC que incluye solamente la vigilancia del cumplimiento de la cláusula de no afectación entre la PESC y otras políticas de la UE (art. 40 TUE) y el control de legalidad de las medidas restrictivas adoptadas contra personas físicas o jurídicas (art. 275.2 TFUE). Sin embargo, esta última disposición no le ha conferido competencia para pronunciarse sobre una acción de reparación del perjuicio supuestamente sufrido como consecuencia de medidas restrictivas. En cambio, el TG se declaró competente para conocer la acción indemnizatoria en relación con los reglamentos 861/2010 y 267/2012 porque ambos instrumentos jurídicos se habían basado en el art. 215 TFUE.

El asunto Bank Refah Kargaran ha generado un gran interés porque ha proporcionado al TJUE una excelente oportunidad para perfilar los contornos de las limitaciones del control judicial de la PESC (Van Elsuwege and De Coninck, 2020; Butler, 2020; Eckes, 2020). El TJUE ha tenido que optar entre conceder prioridad a una interpretación textual de los Tratados en los que no se prevé expresamente la posibilidad de presentar una acción indemnizatoria contra las decisiones PESC o una interpretación teleológica con el objetivo de evitar que se produzcan lagunas en la tutela judicial de los particulares. El TJUE no ha dudado en decantarse por la última interpretación.

El objetivo principal de este trabajo es analizar las implicaciones de la sentencia para el control judicial de la PESC y, en general, para el conjunto del ordenamiento jurídico de la UE. En primer lugar, se examinará el alcance del control judicial de la PESC, tal y como aparece configurado en los Tratados. En segundo lugar, se analizarán los pronunciamientos anteriores del TJUE y del TG en relación con el alcance del control judicial de la PESC. La relevancia que

Consejo, de 23 de marzo de 2012 , relativo a medidas restrictivas contra Irán y por el que se deroga el Reglamento (UE) n. ${ }^{\circ}$ 961/2010 (DO L 88, 24.3.2012, p. 1).

6 Decisión 2013/661/PESC del Consejo, de 15 de noviembre de 2013, por la que se modifica la Decisión 2010/413/PESC relativa a la adopción de medidas restrictivas contra Irán (DO L 306 de 16.11.2013, p. 18); Reglamento de ejecución n. ${ }^{\circ}$ 1154/2013 del Consejo de 15 de noviembre de 2013 por el que se aplica el Reglamento (UE) n. ${ }^{\circ}$ 267/2012 sobre medidas restrictivas contra Irán (DO L 306, de 16.11.2013, p. 3).

7 Sentencia del Tribunal General (Sala Primera) de 30 de noviembre de 2016, T-65/14, Bank Refah Kargaran, EU:T:2016:692. 
presenta el control judicial de la PESC ha conducido a que tanto el TJUE como el TG hayan tenido que intervenir en varias ocasiones en los últimos años para resolver cuestiones de gran importancia para la configuración jurídica política de la PESC. Por último, se analizará el razonamiento seguido tanto por el TJUE como por el abogado general Hogan en sus conclusiones. La sentencia Bank Refah Kargaran tiene profundas implicaciones constitucionales para el ordenamiento jurídico de la UE, ya que viene a confirmar que el rol del TJUE en la PESC no es tan limitado como aparentan los Tratados.

\section{LA EXCLUSIÓN DEL CONTROL JUDICIAL DE LA PESC EN LOS TRATADOS}

Para comprender las implicaciones del pronunciamiento del TJUE en el asunto Bank Refah Kargaran es necesario examinar la configuración del control judicial de la PESC, tal y como aparece previsto en los Tratados ${ }^{8}$. El TUE contempla la competencia del Tribunal en términos muy amplios. El art. 19 prevé que el TJUE «garantizará el respecto del Derecho en la interpretación y aplicación de los Tratados». Sin embargo, el art. 24.1 TUE excluye genéricamente el control judicial de los tribunales de la UE en relación con las disposiciones de derecho primario relativas a la PESC y de los actos jurídicos adoptados por las instituciones. Esta exclusión generalizada ha estado presente en los Tratados desde que se introdujo por primera vez la cooperación política europea en el Acta Única Europea y, posteriormente, la PESC en el Tratado de Maastricht. La exclusión del control judicial de la PESC está específicamente reflejada en el art. 275 TFUE que prevé que «el Tribunal de Justicia de la Unión Europea no será competente para pronunciarse sobre las disposiciones relativas a la política exterior y de seguridad común ni sobre los actos adoptados sobre la base de éstas». Tal y como ha sido señalado, "whilst this

$8 \quad$ El abogado general Wahl señaló en el asunto H. Consejo y Comisión que «es preciso reconocer que, en el ámbito de la PESC, la Unión está facultada para adoptar actos jurídicamente vinculantes no solo respecto a sus instituciones, sino también respecto a los Estados miembros. La redacción de los artículos 24 TUE, apartado 3, y 31 TUE, apartado 1, resulta particularmente esclarecedora a este respecto. Por otro lado, no se prevé que la Unión adopte, en materia de PESC, actos por los que se establecen normas generales y abstractas de las que nacen derechos y obligaciones para las personas. Ello explica, en lo esencial, por qué se concibió la PESC, desde su creación en el Tratado de Maastricht, como un conjunto de normas que yo definiría como lex imperfecta» (Conclusiones en el asunto H. Consejo y Comisión, presentadas el 7 de abril de 2016, EU:C:2016:212, puntos 37 y 38). 
exclusion has been part of the DNA of the Union's foreign and security policy, CFSP matters were not conceived of as hermetically sealed from all other areas of EU law» (Koutrakos, 2018: 6). Por esta razón, la doctrina ha prestado mucha atención a la exclusión del control judicial de la PESC en los últimos años (Hinarejos, 2009; Griller 2013; de Baere and Koutrakos, 2012; Wessel, 2016; Andrés Sáenz de Santamaría, 2017; Hillion, 2016; Wessel, 2018; Santos Vara, 2021). Hillion ha demostrado que el argumento de que el TJUE no dispone de competencia en la PESC no es defendible tras la entrada en vigor del Tratado de Lisboa (Hillion, 2014: 47). En su opinión, el Tratado de Lisboa ha otorgado competencia al TJUE en las siguientes materias:

First, it has made it possible for the Court, albeit within limits, to exercise judicial control with regard to certain CFSP acts, thus abolishing the policy's conventional immunity from judicial supervision. Second, it has recalibrated the Court's role in patrolling the borders between EU (external) competences based on the TFEU and the CFSP, turning it into the guarantor of the latter's integrity. Third, the Treaty has generalized the Court's capacity to enforce the principles underpinning the Union's legal order (Hillion, 2014: 47).

Como consecuencia de los cambios introducidos por el Tratado de Lisboa, no se excluye de forma generalizada el control del TJUE de la PESC. Al contrario, se considera que el Tribunal es competente, salvo que se haya excluido su competencia en situaciones específicas (Hinarejos, 2009: 150). El Tratado de Lisboa ha introducido por primera vez dos excepciones a la exclusión generalizada del control judicial de la PESC. En primer lugar, al TJUE le corresponde la vigilancia del cumplimiento de la cláusula de no afectación mutua entre la PESC y otros ámbitos de actuación de la UE, prevista en el art. 40 TUE. En segundo lugar, los tribunales de la Unión son competentes para controlar la legalidad de las decisiones adoptadas por el Consejo sobre la base de las disposiciones relativas a la PESC por las que se establezcan medidas restrictivas frente a personas físicas o jurídicas. El art. 275 TFEU prevé que:

el Tribunal de Justica será competente para control el respeto del artículo 40 del Tratado de la Unión Europea y para pronunciarse sobre los recursos interpuestos en las condiciones contempladas en el párrafo cuarto del artículo 263 del presente Tratado y relativos al control de la legalidad de las daciones adoptadas por el Consejo en virtud del capítulo 2 del título $V$ del Tratado de la Unión Europea por las que se establezcan medidas restrictivas frente a personas físicas o jurídicas.

La primera excepción a la exclusión del control judicial del TJUE tiene un alcance constitucional. El objetivo es garantizar que las disposiciones que regulan la PESC en el Título V del TEU, por un lado, y las disposiciones del TFUE que 
gobiernan el resto de la acción exterior, por otro lado, sean respetadas por las instituciones. Se trata de proteger tanto las particularidades que presenta la PESC como el resto de la acción exterior de la contaminación o efectos negativos de la PESC. El art. 40 está directamente vinculado con el respecto del principio del equilibrio constitucional previsto en el art. 13.2 TUE (Koutrakos, 2018:7)9. La segunda excepción a la exclusión del control judicial de los actos de la PESC fue introducida por el Tratado de Lisboa para responder a los problemas que generaba la proliferación de las sanciones selectivas en relación con la protección de los derechos fundamentales y el derecho a tutela judicial efectiva (Santos Vara, 2009 y $2011)^{10}$. Las sanciones selectivas se materializan generalmente en medidas restrictivas de carácter económico (como por ejemplo la congelación de los bienes de las personas sospechosas de financiar el terrorismo internacional) que son implementadas a través de actos jurídicos de la UE, o en medidas que afectan a la seguridad (como por ejemplo los embargos de armas o las prohibiciones de entrada en el territorio de los Estados miembros).

\section{EL ALCANCE DEL CONTROL JUDICIAL DE LA PESC EN LA JURISPRUDENCIA DEL TRIBUNAL DE JUSTICIA}

La competencia limitada de los tribunales de la Unión en el ámbito de la PESC ha sido claramente matizada a través de la jurisprudencia del TJUE.

9 En el asunto C-130/10, Parlamento c. Consejo, el TJUE tuvo la oportunidad de desarrollar por primera su interpretación del art. 40 TUE en el período post-Lisboa. Se trataba de determinar la base jurídica correcta de las medidas restrictivas dirigidas contra determinadas personas y entidades asociadas con Usamah bin Ladin, la red $\mathrm{Al}$ Qaeda y los talibanes. El TJUE determinó que las sanciones debían fundamentarse en el art. 215 TFUE y no en el art. 75 TFUE (en el Espacio de Libertad, Seguridad y Justicia), a pesar de que el Parlamento Europeo tiene atribuido un papel más limitado en el marco del art. 215 TFUE. El TJUE se refirió en numerosas ocasiones en su razonamiento a la PESC (Sentencia del Tribunal de Justicia (Gran Sala) de 19 de julio de 2012, Parlamento c. Consejo, C-130/10, EU:C:2012:472).

10 A esta cuestión se refiere también la Conferencia Intergubernamental de 2007 en una declaración anexa a los Tratados en la que se recuerda que se debe prestar «la debida atención a la protección y al respecto del derecho de las personas físicas o entidades de que se trate a disfrutar de las garantías previstas en la ley» (Declaración relativa a los artículos 75 y 215 del Tratado de Funcionamiento de la Unión Europea). De este modo, se ha querido dejar claro que los actos jurídicos en los que se materializan las sanciones contra determinadas personas u organizaciones están sometidas al control judicial de los tribunales de la UE. 
Desde la entrada en vigor del Tratado de Lisboa, el TJUE ha desarrollado una línea jurisprudencial constante que ha contribuido significativamente a reforzar la competencia de los tribunales de la UE en el ámbito de la PESC. El TJUE se enfrentó por primera vez a esta cuestión en el asunto Mauricio, en el que tuvo que determinar si tenía competencia para conocer del alcance de la obligación de información al Parlamento Europeo en relación con los acuerdos referidos exclusivamente a la PESC (art. 218.10 TFUE) ${ }^{11}$. El TJUE reconoció que, en principio, no tiene competencia en relación con las disposiciones referidas a la PESC o con los actos adoptados sobre la base de éstas. Sin embargo, no dudó en afirmar que «una excepción a la regla de la competencia general que el artículo 19 TUE confiere al Tribunal de Justicia para garantizar el respeto del Derecho en la interpretación y aplicación de los Tratados y, en consecuencia, deben interpretarse restrictivamente» ${ }^{12}$. Con independencia de que se trate de acuerdos que versan total o parcialmente sobre la PESC, se trata de acuerdos internacionales de los que es responsable la UE. Por esta razón, no cabe afirmar que el TJUE no pueda ejercer el control judicial sobre los acuerdos internacionales PESC o las partes de otros acuerdos que traten de cuestiones PESC (Wessel, 2016: 459).

Posteriormente, en el asunto Tanzania se plantearon cuestiones jurídicas similares a las examinadas en el asunto Mauricio $^{13}$. Por lo que se refiere al control judicial de la PESC, esta cuestión fue examinada con rigurosidad por la abogada general Kokott, quien afirmó que «el Tribunal de Justicia o tiene la competencia o no la tiene. Las excepciones a su competencia requieren una disposición expresa y se han de interpretar restrictivamente. Ni en el art. 24 TUE, apartado 1, párrafo segundo, sexta frase, ni en el art. 275 TFUE, párrafo primero, se establecen graduaciones respecto a las facultades de los tribunales de la Unión para conocer de recursos de anulación en relación con el art. 40 TUE o con el art. 218 TFUE, apartado 10» ${ }^{14}$. Los asuntos Mauricio y Tanzania han contribuido sustancialmente a mitigar la naturaleza intergubernamental de la PESC que, en principio, estaba al margen de los controles parlamentario y judicial (Santos Vara, 2019: 63 y 2021). Se ha señalado que ambos casos se pueden contemplar «as underlining that CFSP is part and parcel of the Union's constitutional set-up» (Wessel, 2016: 459). La amplia

11 Sentencia del Tribunal de Justicia de 24 de junio de 2014, C-658/11, Parlamento Europeo/Consejo, EU:C:2014:2025.

12 Apdos. 69 y 70 de la sentencia (op. cit. nota 10).

13 Sentencia del Tribunal de Justicia de 14 de junio de 2016, C-263/14, Parlamento Europeo/Consejo, EU:C:2016:435.

14 Conclusiones de la abogada general Kokott de 28 de octubre de 2015 en el asunto Tanzania, EU:C:2015:729, punto 47. 
interpretación de la competencia del TJUE en el ámbito de la PESC no era totalmente inesperada, ya que no se podía establecer una separación nítida entre la PESC y el resto de las políticas exteriores de la UE tras la entrada en vigor del Tratado de Lisboa (Hillion, 2014: 47; Wessel, 2019: 135).

La interpretación del alcance del control judicial de la PESC realizada en los asuntos Mauricio y Tanzania ha sido confirmada y desarrollada en la jurisprudencia posterior del TJUE y del TG. En Elitalina, el TJUE aceptó su competencia para conocer de la interpretación y aplicación de las disposiciones del reglamento financiero de la UE en relación con la adjudicación de un contrato público de servicios por el jefe de la Misión EULEX Kosovo que generó gastos a cargo del presupuesto de la Unión. La exclusión de la competencia del TJUE prevista en los arts. 24.1 TUE y 275 TFUE no implica excluir la competencia del Tribunal para interpretar y aplicar el reglamento financiero en relación con la adjudicación de contratos públicos ${ }^{15}$.

En el asunto $H$ c. Consejo y Comisión, la Gran Sala del TJUE llegó a la misma conclusión que en el asunto Elitalina ${ }^{16}$. Se trataba de un recurso contra varias decisiones relativas a un cambio de destino de una magistrada italiana adoptadas por el jefe de personal de la Misión de Policía de la UE (MPUE) en Bosnia Herzegovina. El razonamiento del TJUE se fundamentó en dos argumentos. En primer lugar, el Tribunal consideró que la existencia misma de un control jurisdiccional efectivo para garantizar el cumplimiento de las disposiciones del derecho de la Unión es inherente a la existencia del Estado de derecho ${ }^{17}$. En segundo lugar, si bien las decisiones controvertidas se inscriben en el contexto de la PESC, el Tribunal estimó que «tal circunstancia no puede llevar necesariamente a excluir la competencia del juez de la Unión» ${ }^{18}$. A pesar de que las decisiones relativas a la asignación de recursos humanos en la zona de operaciones de una misión tienen un carácter operativo en el ámbito de la PESC, «tales decisiones constituyen igualmente, por su propia esencia, actos de gestión de personal, como toda decisión similar adoptada por las instituciones de la Unión en el ejercicio de sus competencias» ${ }^{19}$.

El asunto Rosneft le va a permitir al TJUE adentrarse por primera vez en el núcleo competencial duro del sistema jurisdiccional de la UE en

15 Sentencia del Tribunal de Justicia de 12 de noviembre de 2015, C-439/13 P, Elitalina/ Eulex Kosovo, apdo. 49.

16 Sentencia del Tribunal de Justicia de 19 de julio de 2016, C-455/14 P, H/Consejo y Comisión, EU:C:2016:569.

17 Ibid., apdo. 41.

18 Ibid., apdo. 43.

19 Ibid., apdo. 53. 
relación con la PESC, ya que los pronunciamientos anteriores versaban sobre cuestiones muy específicas $^{20}$ (Andrés Sáenz de Santamaría, 2017: 881). El Tribunal Superior de Justicia (Inglaterra y Gales) en el Reino Unido planteó una cuestión prejudicial sobre la validez de determinadas disposiciones de la Decisión 2014/512/PESC del Consejo, de 31 de julio de 2014, y sobre la validez e interpretación del Reglamento 833/2014 del Consejo, de 31 de julio de 2014. Esta cuestión se suscitó en el marco de un litigio entre Rosneft, una empresa rusa especializada en los sectores petrolíferos y gasísticos, y las autoridades británicas responsables de implementar las sanciones de la UE. En Rosneft, el TJUE aceptó su competencia para conocer de cuestiones prejudiciales de validez en relación con las medidas restrictivas adoptadas contra personas físicas y jurídicas adoptadas en el marco de la PESC ${ }^{21}$. A este respecto, el TJUE afirma que «cuando el art. 24 TUE, apartado 1, párrafo segundo, última frase, remite al art. 275 TUE, párrafo segundo, no lo hace para determinar el tipo de procedimiento en que el Tribunal de Justicia puede controlar la legalidad de determinadas decisiones, sino para fijar el tipo de decisiones cuya legalidad puede ser controlada por el Tribunal de Justicia en cualquier procedimiento cuyo objeto sea ese mismo control de legalidad ${ }^{22}$. A esta línea jurisprudencial consolidada se podrían añadir otros casos, tales como Jenkinson y SanCen c. KF, en los que el TJ ha contribuido a perfilar el alcance del control judicial de los tribunales europeos en el marco de la PESC $^{23}$.

El análisis de la jurisprudencia anterior sobre el control judicial de la PESC a Bank Refah Kargaran nos pone claramente de manifiesto que los

20 Sentencia del Tribunal de Justicia de 28 de marzo de 2017, Rosneft, C-72/15, EU:C: 2017:236.

21 En el asunto Rosneft, el abogado general Whatelet señaló en sus conclusiones que el hecho de que una cuestión esté relacionada con la PESC no supone que sea inmune al control del TJUE. Así, afirmó: «Deseo señalar que la limitación de la competencia del Tribunal de Justicia en materia de PESC operada por la cláusula "carve-out" viene motivada por el hecho de que, en principio, se supone que los actos PESC se limitan a reflejar decisiones de carácter puramente políticas vinculadas al ejercicio de la PESC, cuyo control jurisdiccional es difícilmente conciliable con la separación de poderes» (Conclusiones del abogado general Whatelet, presentadas el 31 de mayo de 2016, EU:C:2016:381, punto 52).

22 Sentencia Rosneft (op. cit., nota 20, apdo. 70).

23 Auto del Tribunal General (Sala Primera) de 9 de noviembre de 2016, Jenkinson/ Consejo y otros, EU:T:2016:660; Sentencia del Tribunal General (Sala Novena ampliada) de 25 de octubre de 2018, KF/Centro de Satélites de la Unión Europea (CSUE), EU:T:2018:718. 
actos jurídicos PESC no pueden ser ya considerados como instrumentos de naturaleza meramente intergubernamental ${ }^{24}$. Los actos de la PESC que no tienen un carácter claramente político puede ser objeto de control judicial por los tribunales de la UE. Se puede deducir de la jurisprudencia del TJUE que los actos de la PESC han de ser interpretados en el marco de la estructura general de los Tratados, incluyendo el sistema general de recursos de la UE. Esta línea jurisprudencial va a resultar claramente consolidada y desarrollada en el asunto Bank Refah Kargaran.

\section{LA OPINION DEL ABOGADO GENERAL HOGAN: LA NECESARIA COHERENCIA DE LOS TRATADOS}

El abogado general Hogan reconoce que la competencia de los tribunales de la Unión en el ámbito de la PESC es limitada. A primera vista, los Tratados no confieren competencia a los tribunales de la Unión para conocer de las acciones indemnizatorias dirigidas a obtener una reparación por los daños sufridos a consecuencia de actos adoptados en el ámbito de la PESC. En cambio, el Tribunal de Justicia dispone de competencia para conocer de los recursos interpuestos por los daños presuntamente sufridos por una persona física o jurídica a causa de la aplicación de medidas restrictivas adoptadas en el marco del art. 215 TFUE. Siguiendo las conclusiones presentadas por el abogado general Wahl en el asunto H/Consejo y Comisión ${ }^{25}$, el abogado general Hogan estima que la limitación del control judicial de la PESC refleja prácticas asentadas de los tribunales nacionales en relación con las decisiones adoptadas en materia de política exterior por los Gobiernos de los Estados miembros. Esta deferencia hacia el poder ejecutivo por los tribunales se explica "porque muchas de estas decisiones [...] tratan asuntos de alta política y diplomacia que, por su propia naturaleza, no son susceptibles de resolución judicial $\aleph^{26}$. Además, es necesario respetar la discrecionalidad política de los Gobiernos de los Estados miembros en relación con las decisiones de política exterior. En definitiva, las cuestiones que se plantean en materia de política exterior

24 Tal y como ha señalado Cardwell, "the perspective of the CFSP as being intergovernmental is not only out-dated but misleading because it stresses that the Member States are the only significant actors in it and that anything which concerns the world beyond the borders of the EU must take place within CFSP» (Cardwell, 2015: 456).

25 Conclusiones del abogado general Wahl, presentadas el 7 de abril de 2016, H/Consejo y Comisión, EU:C:2016:212.

26 Conclusiones del abogado general Hogan, presentadas el 28 de mayo de 2020, C-234/19 P, Bank Refah Kargaran, EU:C:2020:396, punto 47. 
no son susceptibles de ser resueltas aplicando los métodos tradicionales de apreciación de las pruebas y de valoración jurídica (Butler, 2020: 3).

El abogado general Hogan estima que no todos los actos adoptados en el ámbito de la PESC quedan excluidos del control del TJUE por los Tratados ${ }^{27}$. En primer lugar, solamente los actos adoptados sobre la base de una de las disposiciones recogidas en los arts. 23 TUE a 46 TUE están excluidas del control judicial. En segundo lugar, el TJUE ya ha declarado que, por su propia naturaleza, determinados actos no están excluidos del ámbito del control jurisdiccional, como, por ejemplo, la validez de los actos de gestión del personal. En tercer lugar, dado que el art. 24 TUE, apdo. 1, párr. segundo, y el art. 275 TFUE, párrafo primero, introducen una excepción a la regla de la competencia general que el art. 19 TUE confiere al TJUE para garantizar el respeto del derecho en la interpretación y aplicación de los Tratados, deben interpretarse restrictivamente.

A primera vista, el art. 263 TFUE solo contempla la posibilidad de revisar las medidas restrictivas contra personas y entidades en el marco del recurso de nulidad. Sin embargo, esta interpretación fue superada por el pronunciamiento del TJUE en el asunto Rosneft. El abogado general Hogan pone claramente de manifiesto que el art. 275 TFUE no se puede interpretar de manera estrictamente literal. En este sentido, «el Tratado debe leerse de forma integral y armónica, de manera que sus distintos componentes produzcan un resultado que, adaptando los términos utilizados por el Tribunal de Justicia en el apartado 78 de la sentencia Rosneft, garantice "la necesaria coherencia" inherente a todo sistema de protección judicial $»^{28}$. Los autores de los Tratados no excluyeron explícitamente la posibilidad de que se pudiera presentar una acción indemnizatoria por los daños supuestamente sufridos como consecuencia de las medidas restrictivas adoptadas en el marco de la PESC.

\section{EL ENFOQUE DEL TRIBUNAL DE JUSTICIA: UN AVANCE REVOLUCIONARIO}

\section{EL ESTADO DE DERECHO Y LA TUTELA JUDICIAL EFECTIVA: UNA REFERENCIA PREVISIBLE}

En las sentencias H/Consejo y Rosneft, el TJUE fundamentó su razonamiento en el principio del Estado de derecho, al que es inherente la existencia de un control jurisdiccional efectivo. En este sentido, se puso de manifiesto

27 Ibid., punto 50.
28 Ibid., punto 61. 
que el art. 47 de la Carta, que constituye una reafirmación del principio de la tutela judicial efectiva, exige que toda persona cuyos derechos y libertades garantizados por el derecho de la Unión hayan sido violados tenga derecho a la tutela judicial efectiva. En Rosneft, el TJUE estimó que «si bien el art. 47 de la Carta no puede crearle al Tribunal de Justicia una competencia cuando los Tratados excluyen esa posibilidad, el principio de la tutela judicial efectiva conlleva que la exclusión de la competencia del Tribunal de Justicia en materia PESC debe interpretarse restrictivamente» ${ }^{29}$. El abogado general Wathelet prestó especial atención en su argumentación al Estado de derecho, señalando que la posición de que en la PESC los Tratados no otorgan al TJUE la potestad de pronunciarse prejudicialmente es «difícilmente conciliable con el art. 23 TUE», según el cual la acción de la Unión en la escena internacional se basará en los principios del Estado de derecho, y la universalidad e indivisibilidad de los derechos humanos y de las libertades fundamentales, que, sin duda, incluyen el derecho de acceso a la justicia y a la tutela judicial efectiva ${ }^{30}$.

En este contexto, era previsible que el TJUE introdujera referencias a los principios del Estado de derecho y la tutela judicial efectiva en su pronunciamiento en el asunto Bank Refah Kargaran. El TJUE considera que el art. 275 TFUE no reconoce expresamente su competencia para pronunciarse sobre los perjuicios supuestamente sufridos como consecuencia de las medidas restrictivas establecidas en el marco de la PESC. Siguiendo el razonamiento desarrollado en la sentencia Rosneft, el TJUE señala que los arts. 24 TUE y 275 TFUE introducen una excepción a la regla de la competencia general que el art. 19 TUE confiere al Tribunal que debe interpretarse restrictivamente ${ }^{31}$. Si bien la acción prevista para solicitar una indemnización es distinta del recurso de nulidad, debe examinarse en relación con el conjunto del sistema de tutela judicial establecido por los Tratados y, en particular, del derecho a la tutela judicial efectiva. En este sentido, el Tribunal recuerda que la Unión se basa en los principios del Estado de derecho y de la tutela judicial efectiva, lo que implica que toda persona cuyos derechos y libertades garantizados por el derecho de la Unión hayan sido violados tenga derecho a la tutela judicial efectiva.

En una Unión que se fundamenta en el Estado de derecho no debería plantear dudas que las personas incluidas en una lista de sanciones puedan interponer un recurso de indemnización por los perjuicios sufridos. En este

29 Sentencia Rosneft, cit., nota 20, apdo. 74.

30 Conclusiones del abogado general Whatelet en el asunto Rosneft, C-72/15, cit., punto 66.

31 Sentencia Bank Refah Kargaran, cit., apdo. 32. 
sentido, la argumentación seguida por TJUE en el asunto Bank Refah Kargaran enlaza claramente con la sentencia Los Verdes, si bien no es mencionada expresamente $^{32}$. En realidad, el TJUE ha venido aplicando este razonamiento en toda la jurisprudencia posterior a la entrada en vigor del Tratado de Lisboa en la que ha analizado su competencia en el ámbito de la PESC (Van Elsuwege y Joyce De Coninck, 2020: 4). Se ha señalado acertadamente que la doctrina establecida por el TJUE en el asunto Los Verdes sobre un "sistema completo de vías de recurso y de procedimientos» destinado a confiar al Tribunal el control de la legalidad de los actos de las instituciones «is alive and well, and living in current EU external relations case law» (Butler y Wessel, 2021: 5).

\section{LA RELEVANCIA DEL RECURSO DE INDEMNIZACIÓN EN LA PESC Y LA TUTELA JUDICIAL EFECTIVA}

Dado que el TG declinó su competencia para conocer de los perjuicios supuestamente sufridos como consecuencia de las medidas restrictivas adoptadas en el marco de la PESC, el TJUE tuvo que examinar esta cuestión antes de pronunciarse sobre el fondo del recurso planteado por la recurrente. Con su decisión en el asunto Bank Refah Kargaran, el TJUE reconoce sin ambigüedades que las personas físicas y jurídicas pueden interponer un recurso para solicitar una indemnización por los daños sufridos como consecuencia de las medidas restrictivas establecidas en el marco de la PESC. Con anterioridad a Bank Refah Kargaran, esta posibilidad no estaba clara ${ }^{33}$. Como se ha puesto de manifiesto anteriormente, para llegar a este resultado el TJUE contó con las elaboradas conclusiones del abogado general Hogan, quien abogó por una interpretación armónica y coherente de los Tratados.

A la luz de la jurisprudencia previa del TJUE sobre el control judicial de la PESC, no es sorprendente que haya aceptado su competencia para examinar un recurso de indemnización que tenga por objeto obtener la reparación de los daños causados por medidas restrictivas establecidas en el marco de la PESC. Dado que el art. 275.2 TFUE regula una manifestación del derecho a

32 Sentencia de 23 de abril de 1986, C-294/83, Los Verdes c. Parlamento, EU:C:1986:166.

33 En el pasado, el TG había rechazado la competencia de los tribunales de la UE para examinar recursos de indemnización que tenía por objeto reparar los perjucios causados por adoptados en el marco de la PESC. Se pueden mencionar los siguientes asuntos: Sentencia del Tribunal General de 23 de mayo de 2013, Trabelsi y otros, T-187/11, EU:T:2013:273; Auto del Presidente del Tribunal General de 19 de mayo de 2011, Dagher, T-218/11, EU:T:2012:8; Auto del Tribunal de Justicia de 1 de diciembre de 2015, Georgias y otros, T-168/12, EU:C:2015:791; Sentencia del Tribunal General de 18 de febrero de 2016, Jannatian, T-328/14, EU:T:2016:86. 
la tutela judicial efectiva, es comprensible que el TJUE haya confirmado que el control de legalidad de las medidas restrictivas incluya también el derecho a obtener una indemnización por los daños sufridos PESC (Van Elsuwege y De Coninck, 2020: 4; Butler y Wessel, 2021: 5). La declaración de nulidad de la decisión PESC y del reglamento que ha implementado las sanciones no sería suficiente para satisfacer las exigencias derivadas de derecho a la tutela judicial efectiva.

Sin embargo, el Consejo alegó que, dado que los reglamentos basados en el art. 215 TFUE se limitan generalmente a reproducir el contenido de las decisiones PESC, la tutela judicial de las personas físicas o jurídicas está garantizada. El TJUE no acepta que la posibilidad de obtener una indemnización por los perjuicios supuestamente sufridos como consecuencia de los reglamentos adoptados en virtud del art. 215 TFUE sea suficiente para garantizar la tutela judicial efectiva ${ }^{34}$. Es cierto que las decisiones PESC y los reglamentos que las implementan en el marco del art. 215 TFUE no siempre son materialmente idénticos. Es necesario adoptar reglamentos para aplicar las medidas de carácter económico en toda la UE, pero las restricciones a la admisión en el territorio de los Estados miembros suelen recogerse solamente en decisiones PESC.

Si se adoptara la argumentación desarrollada por el Consejo, existiría una laguna en la protección jurídica de las personas sujetas a medidas restrictivas porque no contarían con un recurso de indemnización disponible para obtener una reparación por el oprobio y la desconfianza generados por las sanciones. Por esta razón, el TJUE afirma que el principio de la tutela judicial efectiva de las personas o entidades sujetas a medidas restrictivas exige que el Tribunal «pueda conocer de un recurso de indemnización interpuesto por estas personas o entidades que tenga por objeto obtener la reparación de los daños causados por medidas restrictivas establecidas en decisiones PESC» ${ }^{35}$.

Es preciso señalar que no es completamente cierto que los particulares estarían desprovistos de protección si no se aceptara la posibilidad de interponer un recurso de indemnización. El TJUE no entra a analizar que las restricciones a la admisión en el territorio de los Estados miembros se enmarcan dentro de las competencias de los Estados miembros y son implementadas por los Estados miembros. En estas circunstancias, las personas sujetas a restricciones a la entrada en los Estados miembros podrían interponer un recurso de indemnización en el Estado o Estados que no le hayan permitido entrar en su territorio. Por lo tanto, el TJUE no ofrece un panorama completo de

34 Sentencia Bank Refah Kargaran, cit., apdo. 40.

35 Sentencia Bank Refah Kargaran, cit., apdo. 43. 
los recursos que tienen a su disposición las personas o entidades sujetas a medidas restrictivas. Ahora bien, es preciso reconocer que los perjuicios derivados del oprobio y la desconfianza que conlleva la inclusión en una lista negra habría que dilucidarlos ante los Tribunales de la UE.

\section{LA COHERENCIA DEL SISTEMA DE TUTELA JUDICIAL: UN ARGUMENTO POCO CONVINCENTE}

Lo que realmente sorprende de la sentencia Bank Refah Kargaran es la facilidad con la que el TJUE llega a la conclusión de que dispone de la competencia necesaria para conocer de un recurso de indemnización que tiene por objeto obtener una reparación de los perjuicios causados por medidas restrictivas establecidas en decisiones PESC (Butler, 2020: 2). Se trata de una cuestión que tiene una clara relevancia constitucional. El razonamiento del Tribunal se fundamenta en que «la necesaria coherencia del sistema de tutela judicial establecido por el Derecho de la Unión exige que, para evitar una laguna en la tutela judicial de las personas físicas o jurídicas afectadas, el Tribunal de Justicia de la Unión Europea sea también competente para pronunciarse sobre los perjuicios supuestamente sufridos como consecuencia de medidas restrictivas establecidas en decisiones PESC» ${ }^{36}$.

El TJUE enlaza con el caso Rosneft para integrar en su razonamiento la coherencia del sistema de protección jurisdiccional. En aquel asunto, el Tribunal señaló que «la necesaria coherencia del sistema de protección judicial exige que la facultad de declarar la nulidad de los actos de las instituciones de la Unión, si se plantea ante un órgano jurisdiccional nacional, esté reservada al Tribunal de Justicia en el marco del art. $267 \mathrm{TFUE} \aleph^{37}$. Este principio deriva de la jurisprudencia reiterada del TJUE que tiene su origen en la sentencia Foto-frost ${ }^{38}$.

36 Sentencia Bank Refah Kargaran, cit., apdo. 39.

37 Sentencia Rosneft, cit., apdo. 74.

38 Sentencia de 22 de octubre de 1987, Foto-Frost, 314/85, EU:C:1987:452, apdo. 17. En el Dictamen 2/13, la abogada general Kokott se opuso a la posibilidad de aplicar la jurisprudencia Foto-Frost en el contexto de la PESC alegando que el control de legalidad de la PESC corresponde a los tribunales nacionales porque esta política sigue presentando un carácter intergubernamental (puntos 100 y 101 de la Opinión, EU:C:2014:2475). En cambio, el abogado general Wahl señaló en el asunto H/Consejo que el principio fundamental dimanante de la jurisprudencia Foto-Frost es también aplicable en el ámbito de la PESC. La anulación de un acto de la PESC por un órgano jurisdiccional nacional podría tener repercusiones muy graves para la política exterior de la Unión y de los Estados miembros (EU:C:2016:212, puntos 102 y 103 de las Conclusiones). 
El objetivo fundamental del art. 267 TFUE es garantizar la aplicación uniforme del derecho de la UE por parte de los órganos jurisdiccionales nacionales. Esta conclusión resulta también aplicable a las decisiones PESC mediante las que se imponen medidas restrictivas frente a personas físicas o jurídicas, y respecto de las que los Tratados confieren al TJUE competencia para ejercer el control de legalidad $^{39}$. Por lo tanto, corresponde al TJUE pronunciarse con carácter prejudicial sobre la compatibilidad de los actos adoptados en el marco de la PESC con los Tratados. Como se ha señalado, el TJUE «pone fin a una ambigüedad que podía acabar causado problemas» (Andrés Sáenz de Santamaría, 2017: 898).

Mientras que en el asunto Rosneft era perfectamente comprensible que se recurriera a la jurisprudencia Foto-frost, la referencia que se hace a la coherencia del sistema de tutela judicial en Bank Refah Kargaran no es plenamente convincente. Si corresponde al TJUE conocer de los recursos de nulidad planteados contra las decisiones PESC en las que se imponen medidas restrictivas contras personas o entidades, es lógico que se le atribuya también la competencia para pronunciarse con carácter prejudicial sobre la validez de las mismas decisiones ${ }^{40}$. No es concluyente afirmar que la referencia a la coherencia del sistema de protección judicial permite colmar las lagunas existentes en la tutela judicial de las personas físicas o jurídicas afectadas por las medidas restrictivas. En el caso de que existan lagunas en la protección judicial de los particulares, habrá que superarlas a través de la reforma de los Tratados. En definitiva, la referencia a la coherencia del sistema de tutela judicial es persuasivo en el contexto del control de legalidad, pero no en relación con un recurso de indemnización interpuesto que tenga por objeto obtener la reparación de los daños causados por medidas restrictivas establecidas en decisiones PESC ${ }^{41}$.

El razonamiento del TJUE habría resultado más convincente si hubiera optado simplemente por una interpretación teleológica de los Tratados. De este modo, una vez que los particulares tienen a su disposición la posibilidad de interponer un recurso de nulidad o de beneficiarse de una cuestión prejudicial de validez interpuesta en relación con decisiones PESC en la que se imponen medidas restrictivas, no tendría sentido que se les negará el derecho

39 A este respecto, el TJUE señaló en la sentencia Rosneft que «las divergencias entre los órganos jurisdiccionales nacionales de los Estados miembros sobre la validez de los actos de la Unión pueden llegar a comprometer la unidad misma del ordenamiento jurídico de la Unión y vulnerar la exigencia fundamental de la seguridad jurídica» (sentencia Rosneft, cit., apdo. 80).

40 Sentencia Bank Refah Kargaran, cit., apdo. 78.

41 Sentencia Bank Refah Kargaran, cit., apdo. 81. 
a plantear un recurso de indemnización para obtener una reparación de los daños causados por medidas restrictivas establecidas en las mismas decisiones PESC.

\section{LA INAPLICACIÓN DE LA JURISPRUDENCIA SEGI Y GESTORAS PRO-AMNISTÍA}

El TJUE descarta la aplicación de la jurisprudencia Segi y Gestoras Pro-Amnistía en el contexto actual ${ }^{42}$. El Consejo alegó que debía seguirse la misma interpretación respecto de la PESC que había seguido el Tribunal en su versión anterior al Tratado de Lisboa. En 2007, el TJUE estimó que el antiguo art. 35 TUE no le otorgaba competencia para conocer de ningún tipo de recurso de indemnización en relación con el tercer pilar (disposiciones relativas a la cooperación policial y judicial en materia penal). Por ello, los órganos jurisdiccionales nacionales eran los únicos competentes para examinar los recursos de indemnización que se pudieran plantear en relación con las disposiciones del segundo y tercer pilares ${ }^{43}$.

Es convincente que el TJUE haya rechazado la aplicación de la jurisprudencia Segi y Gestoras Pro-Amnistía en el asunto Bank Refah Kargaran. No tiene sentido examinar el alcance de las competencias actuales del TJUE en el ámbito de la PESC a la luz de las disposiciones del TUE relativas a esta institución aplicables antes de la entrada en vigor del Tratado de Lisboa. No sería lógico que se interpretaran las disposiciones de la PESC de forma aislada sin tener en cuenta la evolución de los Tratados (Van Elsuwege y De Coninck, 2020: 4). La estructura de los Tratados ha cambiado sustancialmente respecto de la que existía en el momento en que se produjeron los hechos sobre los que versaban las sentencias Segi y Gestoras Pro-Amnistía. La UE se ha dotado de una personalidad jurídica única, tal y como aparece reflejado en el art. 47 TUE, lo que ha supuesto la integración de las disposiciones relativas a la PESC en el ordenamiento jurídico de la Unión, si bien está sujeta a reglas y procedimientos específicos.

42 Sentencias de 27 de febrero de 2007, Gestoras Pro Amnistía y otros/Consejo (C-354/04 P, EU:C:2007:115), apdo. 46, y de 27 de febrero de 2007, Segi y otros/Consejo (C-355/04 P, EU:C:2007:116), apdo. 46.

43 Esta jurisprudencia dejó una cierta sensación de insatisfacción, al remitir a los órganos jurisdiccionales nacionales la competencia para reparar los eventuales daños derivados de las medidas adoptadas por las instituciones de la Unión en el marco de los pilares intergubernamentales (Santos Vara, 2008: 19). 


\section{LA DIFICULTAD PARA OBTENER UNA REPARACIÓN POR LOS PERJUICIOS SUFRIDOS EN EL ORDENAMIENTO JURÍDICO DE LA UE}

Una vez admitida la competencia del Tribunal para pronunciarse sobre la pretensión de reparación del perjuicio sufrido como consecuencia de medidas restrictivas adoptadas en el marco de la PESC, no quiere decir que se vaya a conceder fácilmente la indemnización solicitada. El TJUE enlaza en este aspecto con el razonamiento seguido por el TG y considera que «la motivación insuficiente de un acto por el que se adopta una medida restrictiva no puede, como tal, generar la responsabilidad extracontractual de la Unión ${ }^{44}$. Para que la UE incurra en responsabilidad extracontractual deben cumplirse tres requisitos: primero, debe existir una infracción suficientemente caracterizada de una norma jurídica que tenga por objeto conferir derechos a los particulares; segundo, debe haber un perjuicio, y tercero, ha de existir una relación de causalidad entre la infracción de la obligación que incumbe al infractor y el daño sufrido por las víctimas ${ }^{45}$. Es realmente difícil que se cumpla el primer requisito porque las decisiones del Consejo relativas a la imposición de medidas restrictivas vienen determinadas por consideraciones políticas (Butler y Wessel, 2021: 6). El TJUE estima que la motivación insuficiente o falta de motivación puede generar la responsabilidad de la UE cuando el Consejo no aporta pruebas que permitan acreditar la procedencia de la información ${ }^{46}$.

El Consejo se enfrenta a veces a dificultades para poder disponer de la información que justifica la adopción de medidas restrictivas. Por lo tanto, si no existe un fundamento sólido que justifique las sanciones, se cumpliría el primer requisito para que la Unión pueda incurrir en responsabilidad internacional. El abogado general Hogan pone claramente de manifiesto en sus conclusiones que cuando las medidas restrictivas no se basan en información o pruebas relevantes, se puede generar la responsabilidad de la $\mathrm{UE}^{47}$. El único asunto vinculado al ámbito de las medidas restrictivas en el que se concedió una indemnización al recurrente por los perjuicios sufridos como consecuencia de las medidas restrictivas ha sido en el caso Safa Nicu/Consejo ${ }^{48}$. El TJUE señala que, aunque los hechos de aquel caso y del presente asunto son similares, la indemnización se fundamentó en un error de apreciación,

44 Sentencia Bank Refah Kargaran, cit., apdo. 62.

45 Véase la Sentencia de 4 de julio de 2000, Bergaderm y Goupil/Comisión, C-352/98 P, EU:C:2000:361, apdo. 42.

46 Sentencia Bank Refah Kargaran, cit., apdo. 65.

47 Conclusiones del abogado general Hogan, cit., apdo. 65.

48 Sentencia de 30 de mayo de 2017, Safa Nicu Sepahan/Consejo, C-45/15 P, EU:C:2017:402 
y no, únicamente en una falta de motivación. Por otra parte, no deja de ser paradójico que se pueda exigir la responsabilidad extracontractual de la UE por un error que es imputable al comité de sanciones correspondiente, ya que la UE se limita a implementar las sanciones impuestas por el Consejo de Seguridad y no dispone de margen de discrecionalidad.

\section{REFELEXIONES FINALES}

La sentencia dictada por el TJUE en el asunto Bank Refah Kargaran va a contribuir significativamente a reforzar la protección jurídica de los particulares en relación con las medidas restrictivas adoptadas por la UE. Por primera vez, el TJUE se ha declarado competente para conocer de los recursos que tiene por objeto obtener una indemnización por los perjuicios sufridos como consecuencia de la adopción de medidas restrictivas en el ámbito de la PESC. No es sorprendente que el TJUE haya estimado que la mera declaración de nulidad de las medidas restrictivas adoptadas en el marco de la PESC no es suficiente para satisfacer las exigencias derivadas del derecho a la tutela judicial efectiva, previsto en el art. 47 de la Carta. El Tribunal ha llegado acertadamente a la conclusión de que el art. 275.2 TFUE incluye un aspecto de la tutela judicial efectiva que ha de ser complementado con la posibilidad de presentar un recurso de indemnización para obtener una reparación por el perjuicio derivado de unas medidas que han sido previamente declaradas ilegales. Por lo tanto, todo el sistema de recursos de la UE está a disposición de las personas físicas o jurídicas contra las que se dirigen las medidas restrictivas. El Consejo será probablemente más cauteloso en el futuro a la hora de imponer sanciones por períodos prolongados en el tiempo porque existe el riesgo de que los particulares tengan éxito en los recursos que interpongan para obtener una indemnización por los perjuicios sufridos (Butler y Wessel, 2021: 6).

Si bien la argumentación desarrollada por el TJUE en su pronunciamiento está centrada exclusivamente en las medidas restrictivas establecidas en decisiones adoptadas en el marco de la PESC, la doctrina ha señalado que el derecho a la tutela judicial efectiva, incluido también el recurso de indemnización, podría ser invocado en el contexto de las misiones de gestión de crisis establecidas en el marco de la Política Común de Seguridad y Defensa (PCSD) en el caso de que se produzcan violaciones de los derechos humanos (Van Elsuwege y Joyce De Coninck, 2020: 5; Butler y Wessel, 2021: 6). El TJUE ha levantado el velo sobre la competencia del TJUE para examinar la responsabilidad extracontractual de la UE en el ámbito de la PESC y se 
podrían plantear reclamaciones en el marco de la PCSD en el futuro. Esta cuestión ha de ser clarificada por el TJUE en el futuro.

No está aún claro si la interpretación expansiva del control judicial de la PESC va a tener efectos más allá de las medidas restrictivas dirigidas a personas o entidades. Es preciso reconocer que la gran mayoría de las decisiones PESC no afectan a la situación jurídica de los particulares ${ }^{49}$. La jurisprudencia del TJUE está contribuyendo a superar la exclusión genérica del control judicial de la PESC. Con independencia de que la jurisprudencia del TJUE en relación con el control judicial de la PESC tenga o no efectos más allá de las medidas restrictivas, no cabe duda que el Tribunal está contribuyendo a normalizar la PESC en el ordenamiento jurídico de la UE en la etapa post-Lisboa. A la luz de la evolución de la jurisprudencia del TJUE, los actos jurídicos de la PESC no pueden ser ya considerados como instrumentos de naturaleza netamente intergubernamental que se mantienen al margen del control judicial. La sentencia Bank Refah Kargaran se enmarca en la tendencia del TJUE a asegurar en la medida de lo posible que los actos de la PESC que no tienen un carácter claramente político puedan ser objeto de control judicial por los tribunales de la UE.

Un ámbito en el que previsiblemente el asunto Bank Refah Kargaran va a tener un impacto es en las negociaciones sobre la adhesión de la UE al Convenio Europeo de Derechos Humanos (CEDH), que se han retomado

49 No deja de ser paradójico que el Tribunal Supremo considere que las obligaciones derivadas de las decisiones PESC tienen una naturaleza esencialmente política. El 26 de noviembre de 2020, se pronunció en relación con la entrada en el espacio aéreo español y estancia en el Aeropuerto de Barajas de Delcy Eloína Rodríguez, vicepresidenta de la República Bolivariana de Venezuela. Su entrada en el territorio de los Estados miembros estaba prohibida por la Decisión PESC 2017/2074 del Consejo de la Unión Europea, de 13 de noviembre de 2017, relativa a las medidas restrictivas acordadas en relación con la situación política en Venezuela. El Tribunal Supremo no duda en afirmar que «su incumplimiento implica la vulneración de una obligación en el ámbito de la política exterior de la Unión Europea. El control de su vigencia y su fiscalización incumbe al propio Consejo». El Tribunal Supremo estima también que «el carácter político de estas decisiones se percibe con más nitidez, si cabe, a la vista de los arts. 24 del Tratado de la Unión Europea y el artículo 275 del Tratado de Funcionamiento de la Unión Europea. Ambos preceptos limitan incluso la competencia del Tribunal de Justicia de la Unión Europea en materia de política exterior y de seguridad común». En definitiva, el Tribunal Supremo no solo parece desconocer que las decisiones adoptadas en el marco de la PESC son vinculantes, sino que deja también de lado toda la jurisprudencia del TJUE relativa al control judicial de la PESC (Auto del Tribunal Supremo de 26 de noviembre de 2020, causa especial n.o 20084/2020). 
recientemente. La inexistencia de control judicial de la PESC fue uno de los obstáculos que encontró el TJUE en el controvertido Dictamen 1/13. Mientras que en el Dictamen 1/13 el TJUE señaló que aún no había tenido ocasión de precisar el alcance de las limitaciones de su competencia en el ámbito de la PESC, el examen de la jurisprudencia del TJUE nos pone de manifiesto que su rol es más amplio de lo que se deduce de una lectura literal del art. 24 TUE.

Se podría alegar que, si la intención de los autores de los Tratados era restringir la intervención del TJUE en la PESC, esta limitación no se podría superar a través de la interpretación llevada a cabo por el Tribunal. Además, si los Estados miembros han querido limitar la competencia del TJUE en la PESC, sería necesario reformar los Tratados para ampliarla y no se podría superar a través del activismo judicial del TJUE. En realidad, el TJUE se ha enfrentado a un conflicto entre el argumento de que no dispone de competencia en el ámbito de la PESC y la necesidad de garantizar la tutela judicial efectiva siempre que sea posible. El TJUE no ha dudado en decantarse por la segunda deduciendo su competencia de forma implícita y guiándose en esta labor por los principios y valores en los que se fundamenta la UE. La jurisprudencia del TJUE sobre el control judicial de la PESC confirma que esta política se ha constitucionalizado en gran medida en el ordenamiento jurídico de la UE.

\section{Bibliografía}

Andrés Sáenz de Santamaría, P. (2017). Mejorando la lex imperfecta: tutela judicial efectiva y cuestión prejudicial en la PESC. Revista de Derecho Comunitario Europeo, 58, 871-903. Disponible en: https://doi.org/10.18042/cepc/ rdce.58.02.

Butler, G. (2020). Non-contractual liability and actions for damages regarding restrictive measures through CFSP Decisions: Jurisdiction of the CJEU confirmed. EU Law Live.

Butler, G. y Wessel, R. A. (2021). Jurisdiction of the Court for non-contractual liability and actions for damages claims within the CFSP: Bank Refah Kargaran. En G. Butler y R. Wessel (eds.). EU External Relations Law: The Cases in Context. Oxford: Hart Publishing.

Cardwell, P. J. (2015). On 'Ring-Fencing' the Common Foreign and Security Policy in the Legal Order of the European Union. Northern Ireland Legal Quarterly, 64, 443-463.

De Baere, G. y Koutrakos, P. (2012). The Interactions between the Legislature and the Judiciary in EU External Relations. En P. Syrpis (ed.). The Judiciary, the Legisla- 
ture and the EU Internal Market (pp. 243-273). Cambridge: Cambridge University Press. Disponible en: https://doi.org/10.1017/CBO9780511845680.014.

Denza, E. (2002). The Intergovernmental Pillars of the European Union. Oxford: Oxford University Press. Disponible en: https://doi.org/10.1093/acprof:oso/9780198299356.001.0001.

Eckes, C. (2020). Constitutionalising the EU Foreign and Security Policy: The ECJ accepts jurisdiction over claims for damages under the Common Foreign and Security Policy (CFSP). Verfassungsblog on matters constitutional [blog], 18-102020. Disponible en: https://bit.ly/3aTvSII.

Griller, S. (2013). The Court of Justice and the Common Foreign and Security Policy. En A. Rosas et al. (eds.). Court of Justice of the European Union - Cour de Justice de l'Union Européenne, The Court of Justice and the Construction of Europe: Analyses and Perspectives on Sixty Years of Case-law - La Cour de Justice et la Construction de l'Europe: Analyses et Perspectives de Soixante Ans de Jurisprudence (pp. 675-692). The Hague: T.M.C. Asser Press. Disponible en: https:// doi.org/10.1007/978-90-6704-897-2_36.

Hillion, C. (2014). A Powerless Court? The European Court of Justice and the Common Foreign and Security Policy. En C. Cremona y A. Thies (eds). The European Court of Justice and External Relations Law. Oxford: Hart Publishing. Disponible en: https://doi.org/10.2139/ssrn.2388165.

Hinarejos, A. (2009). Judicial Control in the European Union-Reforming Jurisdiction in the Intergovernmental Pillars. Oxford: Oxford University Press. Disponible en: https://doi.org/10.1093/acprof:oso/9780199569960.001.0001.

Koutrakos, P. (2018). Judicial Review in the EU's Common Foreing and Security Policy. International Comparative Law Quartely, 67, 1-35. Disponible en: https://doi.org/10.1017/S0020589317000380.

Santos Vara, J. (2021). The 'Normalisation' of CFSP International Agreements in the EU Legal Order: Mauritius and Tanzania. En G. Butler y R. Wessel (eds.). EU External Relations Law: The Cases in Context. Oxford: Hart Publishing.

Santos Vara, J. (2019). The European Parliament in the Conclusion of International Agreements Post-Lisbon: Entrenched Between Values and Prerogatives. En J. Santos Vara y S. R. Sánchez-Tabernero (eds.). The Democratization of EU International Relations Through EU Law. Routledge. Disponible en: https://doi. org/10.4324/9781315178721-4.

Santos Vara, J. (2011). The Consequences of Kadi: Where the Divergence of Opinion between EU and International Lawyers Lies? European Law Journal, 17 (2), 252-274. Disponible en: https://doi.org/10.1111/j.1468-0386.2010.00547.x.

Santos Vara, J. (2009). Judicial control over the sanctions against Al-Qaeda and the Taliban in the European Union: a challenge to the powers of the Security Council?. Revista de Derecho Comunitario Europeo, 32, 91-120.

Santos Vara, J. (2008). El control judicial de la ejecución de las sanciones del Consejo de Seguridad en la Unión Europea. Revista Electrónica de Estudios Internacionales, $15,1-23$. 
Van Elsuwege, P. y De Coninck J. (2020). Action for damages in relation to CFSP decisiones pertaining to restrictive measures: A revolutionary move by the Court of Justice in Bank Refah Kargaran? EU Law Analysis, 9-10-2020. Disponible en: http://hdl.handle.net/1854/LU-8677757.

Wessel, R. A. (2019). Legal aspects of parliamentary oversight in EU foreign and security policy. En J. Santos Vara and S. R. Sánchez-Tabernero, (eds.), The democratization of EU international relations through EU law. London: Routledge. Disponible en: https://doi.org/10.4324/9781315178721-4.

Wessel, R. A. (2018). The Good, the Bad and the Ugly: Three Levels of Judicial Control over the CFSP. En S. Blockmans y P. Koutrakos (eds.). Research Handbook in EU Common Foreign and Security Policy (pp. 65-87). Cheltenham; Northampton: Edward Elgar Publishing. Disponible en: https://doi.org/10.4 337/9781785364082.00011.

Wessel, R. A. (2016). Lex Imperfecta: Law and Integration in European Foreign and Security Policy. European Papers: A Journal on Law and Integration, 1 (2), 439-468. 